\title{
Avaliação radiográfica da diplasia coxofemoral de cães adultos: comparação entre dois métodos
}

\author{
[Evaluation of radiographic hip dysplasia in adult dogs: comparison \\ between two methods] \\ B.D. Rocha ${ }^{1}$, R.C.S. Tôrres ${ }^{2}$, E.F. Silva ${ }^{1}$, F.G. Miranda ${ }^{1}$ \\ ${ }^{1}$ Aluno de pós-graduação - Escola de Veterinária - UFMG - Belo Horizonte, MG \\ ${ }^{2}$ Escola de Veterinária - UFMG - Belo Horizonte, MG \\ RESUMO
}

\begin{abstract}
O objetivo deste estudo foi comparar a eficiência dos métodos radiográficos convencional e em distração de cães da raça Pastor Alemão adultos. Foram utilizados 16 animais, sendo onze fêmeas e oito machos, com idade média de quatro anos e dez meses. Todos os cães participantes foram avaliados clinicamente para se detectar a presença de alterações relacionadas ao aparelho locomotor e/ou sistema nervoso que afetariam a locomoção normal do cão, excluindo-se as alterações clínicas decorrentes do quadro de displasia coxofemoral (DCF). Para avaliação radiográfica das articulações coxofemorais, foram realizadas duas técnicas de avaliação, o Método Radiográfico Convencional (MRC), adotado pela Fédération Cynologique Internationale, e o Método Radiográfico em Distração (MRD). Dos animais avaliados, 62,5\% foram classificados com algum grau de DCF. A correlação entre o MRC e o MRD foi moderada e a utilização isolada do MRD, pouco eficaz, com sensibilidade de $75 \%$ e especificidade de $44 \%$. Nas condições em que este experimento foi conduzido, pode-se concluir que o MRC é um exame eficaz no diagnóstico quando comparado ao MRD em cães da raça Pastor Alemão adultos.
\end{abstract}

Palavras-chave: cães adultos, ângulo de Norberg, displasia coxofemoral, índice de distração

\begin{abstract}
The aim of this study was to compare the efficacy of conventional and distraction radiographic methods in adult German Shepherd dogs. Sixteen animals were tested, eleven females and eight males, with a mean age of 4.8 years. All dogs were clinically evaluated to detect locomotor and/or nervous system changes that could affect normal locomotion, but clinical hip dysplasia (HD) alterations were excluded. Two techniques were used for radiographic evaluation of hip joints, the Conventional Radiographic Method (CRM), adopted by Fédération Cynologique Internationale and the Distraction Radiographic Method (DRM). 62.5\% of the evaluated animals were classified as having some degree of HD. The correlation between CRM and DRM was moderate. When DRM was used alone, it was considered an ineffective method, with $75 \%$ sensitivity and $44 \%$ specificity. From this study, it can be concluded that CRM is an effective diagnostic method for hip dysplasia when compared to DRM in adult German Shepherd dogs.
\end{abstract}

Keywords: adult dog, distraction index, hip dysplasia, Norberg angle

\section{INTRODUÇÃO}

A Displasia Coxofemoral (DCF) é a uma anormalidade do desenvolvimento entre a cabeça do fêmur e o acetábulo (Tôrres, 1993). Essa máformação se manifesta por meio da combinação

Recebido em 30 de agosto de 2013

Aceito em 3 de outubro de 2014

E-mail: brunodivino@yahoo.com.br entre o genótipo do indivíduo e fatores ambientais aos quais ele foi exposto durante sua fase de desenvolvimento. A expressão gênica é traduzida em frouxidão da articulação coxofemoral e incongruência desta, desencadeando alterações biomecânicas que, por sua vez, culminam na doença articular 
degenerativa (DAD) (Tôrres et al., 2003; Risler et al., 2009).

Atualmente a DCF é a desordem musculoesquelética não traumática mais comum em cães, podendo representar 30\% do total de casos ortopédicos (LaFound et al., 2002; Souza et al., 2011; Smith et al., 2012). Algumas raças de cães são mais acometidas, como, por exemplo, a Bulldog Inglês, a Pastor Alemão, a Fila Brasileiro, entre outras de médio e grande portes (Lafound et al., 2002; Orthopedic..., 2012). As alterações morfológicas observadas nesses animais são a subluxação ou luxação coxofemoral, o arrasamento do acetábulo, o achatamento da cabeça femoral e outros sinais de DAD (Morgan, 1997; Risler et al., 2009).

Os principais e mais utilizados métodos de diagnóstico da DCF são os radiográficos, dentre os quais se destaca a técnica proposta por Brass e colaboradores (1978), conhecida como Método Radiográfico Convencional (MRC) e adotada pela Fédération Cynologique Internationale (FCI) e pelo Colégio Brasileiro de Radiologia Veterinária (CBRV) (Brass et al., 1978; Colégio..., 2007; Orthopedic...,). Apesar de amplamente utilizada em todo o mundo, essa técnica apresenta um elevado número de resultados falsos negativos quando realizada antes dos 24 meses de idade, fato que prejudica o controle da DCF (Smith et al., 1990; Smith et al., 2002; Tôrres et al., 2005; Froes et al., 2009). Para melhorar a sensibilidade e especificidade dos métodos radiográficos, foram desenvolvidos exames com a articulação coxofemoral sob estresse, denominado Método Radiográfico em Distração (MRD). Eles permitem a deteç̧ão precoce da frouxidão articular e, consequentemente, do risco de desenvolvimento da DCF e DAD (Smith et al., 1990; Risler et al., 2009).

Entretanto são escassos os estudos que mostram a utilização e a eficiência do MRD em cães adultos. O propósito deste experimento foi comparar a eficiência dos métodos radiográficos convencional e em distração de cães da raça Pastor Alemão adultos.

\section{MATERIAL E MÉTODOS}

Foram utilizados 16 cães da raça Pastor Alemão, sendo onze fêmeas e oito machos, com idade média de quatro anos e dez meses. Os animais foram avaliados clinicamente para se detectar a presença de alterações relacionadas ao aparelho locomotor e/ou sistema nervoso que afetariam a locomoção normal do cão, excluindo-se as alterações clínicas decorrentes do quadro de DCF.

Para realização do MRC, os animais foram posicionados em decúbito dorsal, com os membros pélvicos estendidos, rotacionados internamente de modo que a patela permanecesse sobreposta em relação ao plano sagital do fêmur, paralelos entre si e em relação à coluna vertebral e à pélvis em simetria (Fig.1) (Tôrres, 2003).

Para obtenção das imagens radiográficas no MRD, os animais foram colocados em decúbito dorsal com os membros pélvicos em posição neutra em relação à articulação coxofemoral (entre $10^{\circ}$ de flexão e $30^{\circ}$ de extensão, $10^{\circ}$ e $30^{\circ}$ de abdução, e $0^{\circ}$ e $10^{\circ}$ de rotação externa) e os joelhos ficaram fletidos formando um ângulo de $90^{\circ}$ entre o fêmur e a tíbia/fíbula. Depois de o animal ser corretamente posicionado, o Distrator Articular modelo EV-UFMG ${ }^{\circledR}$ foi ajustado entre os membros pélvicos, de forma que suas barras fossem apoiadas na face interna das coxas, próximo à pélvis (Fig. 2 e 3). Os fêmures foram aduzidos para que as cabeças femorais fossem forçadas a saírem do acetábulo.

Para a realização das imagens radiográficas, a quilovoltagem utilizada variou de 72 a $76 \mathrm{kV}$, miliamperagem em 200mA, com um tempo de exposição entre 0,1 e 0,15 de segundo, dependendo do volume de massa muscular dos cães. O foco foi posicionado a $100 \mathrm{~cm}$ das articulações coxofemorais. Para a aquisição das imagens radiográficas, foram utilizados cassetes radiográficos $\mathrm{CR}$ de fósforo (Cassete Reguis Model 170 - Konica Minolta Medical Imaging EUA, Inc.), com 14 x 17 in (35,56 x 43,18cm). 


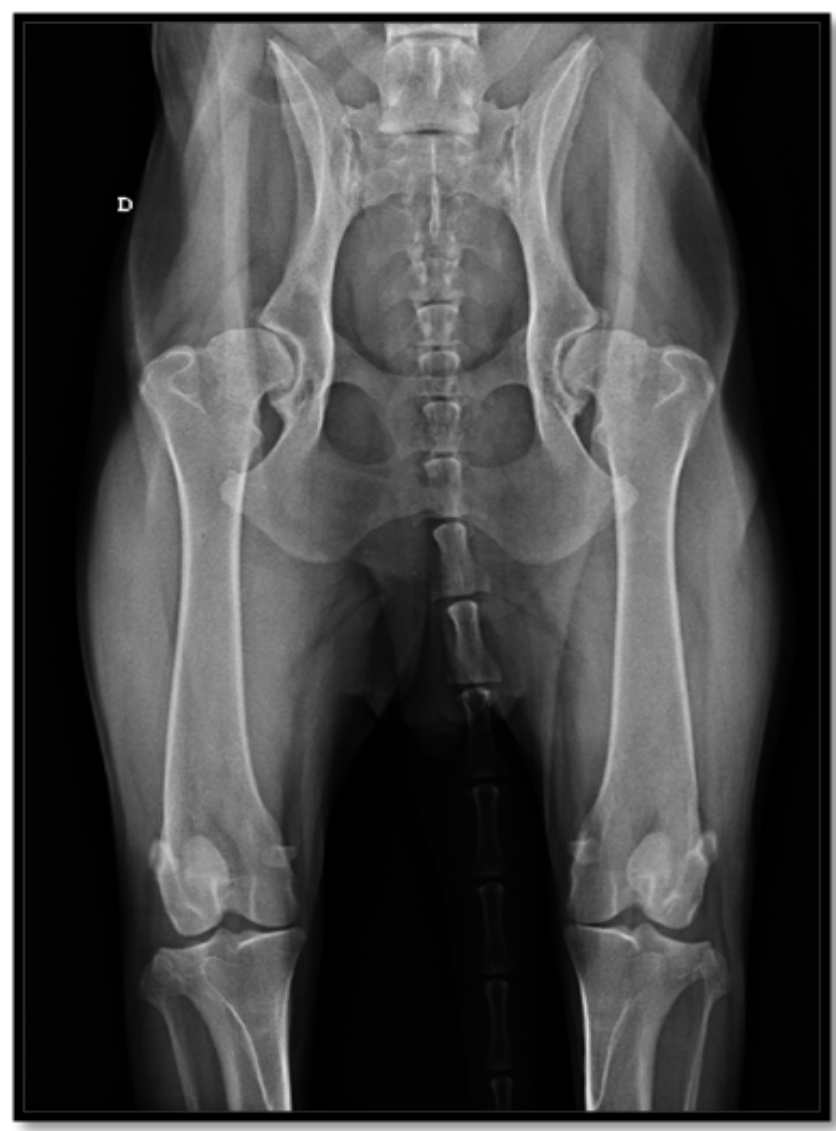

Figura 1. Imagem radiográfica digitalizada das articulações coxofemorais de cão da raça Pastor Alemão, com displasia coxofemoral grave, posicionado para o Método Radiográfico Convencional.

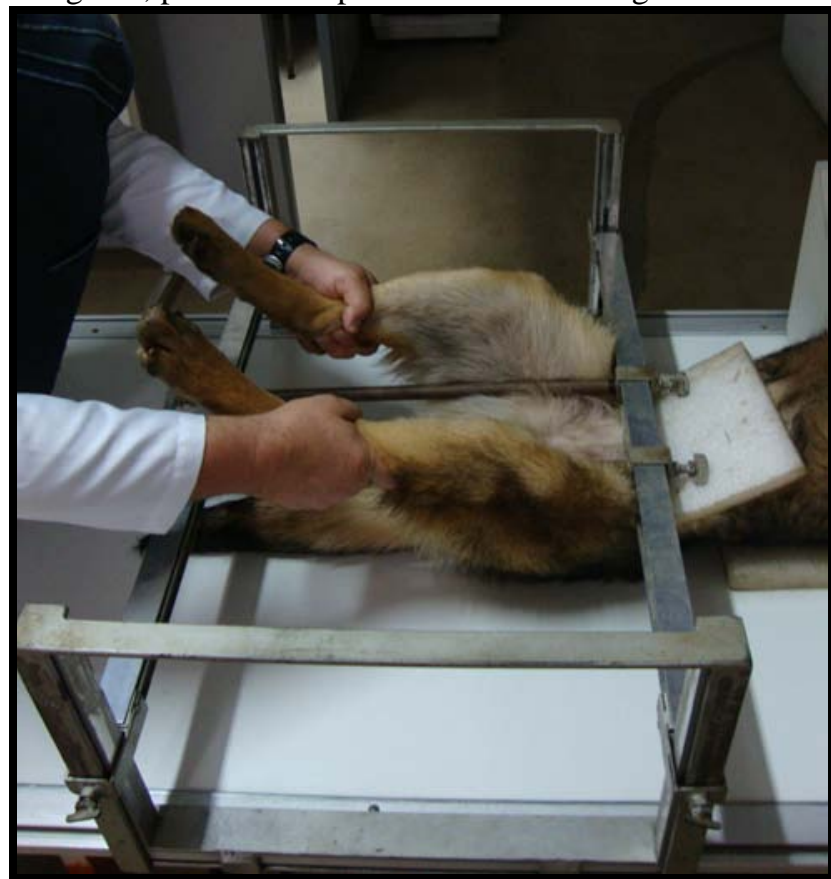

Figura 2. Posicionamento do cão da raça Pastor Alemão, para realização do método radiográfico em distração, utilizando Distrator Articular modelo EV-UFMG ${ }^{\circledR}$. 


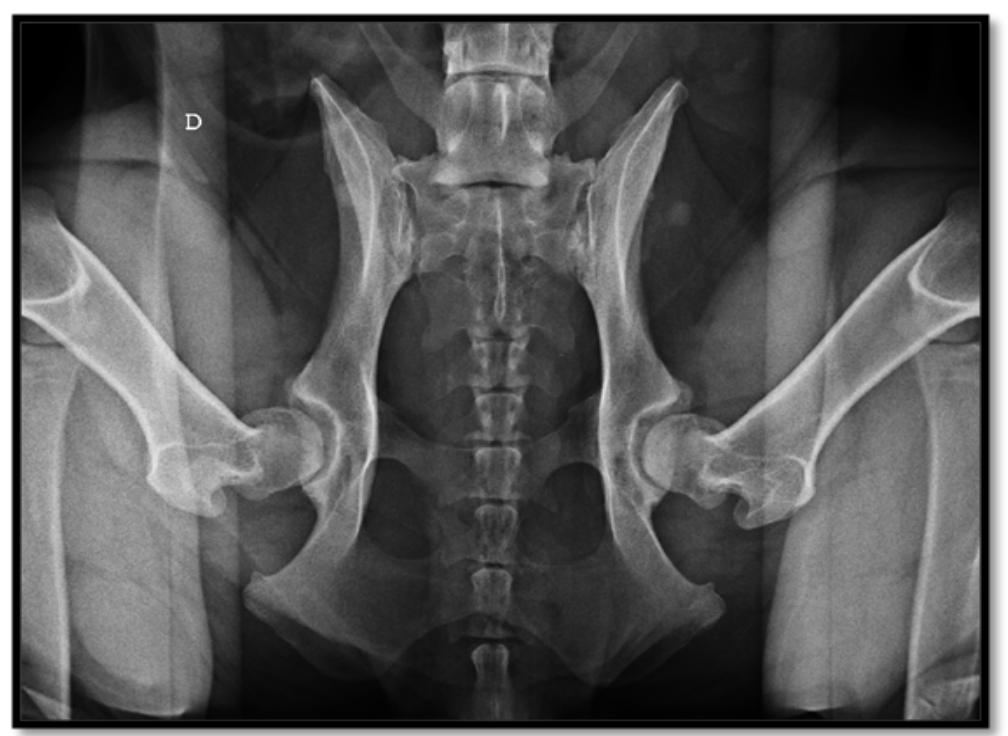

Figura 3. Imagem radiográfica digitalizada das articulações coxofemorais de cão da raça Pastor Alemão, com Índice de Distração < 0,3, posicionado para o Método Radiográfico em Distração, e obtida com o uso do Distrator Articular modelo EV-UFMG ${ }^{\circledR}$.

A avaliação da relação entre a cabeça do fêmur e o acetábulo, nas imagens radiográficas, seguiu o protocolo sugerido por Brass e colaboradores (1978), obtendo-se o ângulo de Norberg, e o cálculo do índice de distração, conforme Smith (1990), as medidas foram obtidas através do software de análise de imagens EMR Manager (EMR Manager versão 7.2 - Vepro AG Germany). Para cada imagem radiográfica analisada, as medições foram repetidas três vezes, sendo consideradas as médias obtidas. A avaliação qualitativa das imagens radiográficas foi realizada conforme classificação proposta por Brass e colaboradores (1978). Com o intuito de reduzir o erro experimental, tanto o posicionamento radiográfico de todos os cães participantes como a interpretação das imagens obtidas foram realizados sempre pelo mesmo examinador. Não foi realizada a comparação das avaliações radiográficas inter observadores ou intra observadores, a utilização da ferramenta digital para medição dos parâmetros suprime o principal ponto de variação entre os resultados inter observadores, a identificação do ponto central da cabeça femoral; dessa forma, a repetibilidade dos valores obtidos é superior a 95\% (Ginja et al., 2006; Froes et al., 2009).ew

A contenção dos animais radiografados seguiu o protocolo de sedação com sulfato de atropina na dose de $0,044 \mathrm{mg} / \mathrm{kg}$, via subcutânea. Após 10 minutos foi administrado cloridrato de xilazina $(0,5 \mathrm{mg} / \mathrm{kg})$ via intramuscular, e 10 minutos depois aplicou-se diazepam $(0,5 \mathrm{mg} / \mathrm{kg})$ via intravenosa. Todos os procedimentos foram aprovados pelo Comitê de Ética em Experimentação Animal da Universidade Federal de Minas Gerais (CETEA/UFMG), registrados no protocolo $n^{\circ} 78 / 2010$.

Considerando que todos os cães do experimento têm idade superior a 24 meses, o MRC foi o padrão ouro de diagnóstico da DCF, por sua vez, nessa idade, é possível detectar alterações radiográficas da DAD na mesma imagem utilizada para aferição do AN. A partir dessa informação, foi calculada a sensibilidade e a especificidade do MRD. A análise estatística foi realizada de maneira a se medir o grau de correlação entre as variáveis estudadas, pelos métodos de correlação de Spearman e análise de variância, por meio da ANOVA. Para todas as análises utilizou-se nível de significância de 0,05 e intervalo de confiança de 95\%. As análises foram realizadas no software SPSS 16.0.

\section{RESULTADOS E DISCUSSÃO}

Dos animais avaliados, 62,5\% foram classificados com algum grau de DCF, seguindo os critérios do CBRV. Esse resultado é semelhante aos observados por Vieira e colaboradores (2010), Tôrres (2003), Tôrres e 
colaboradores (1999 ${ }^{b}$ ) e Tôrres (1993). Contudo, a prevalência foi superior à divulgada pela Orthopedic... (2012) em seu mais recente levantamento, no qual $19 \%$ das radiografias avaliadas foram qualificadas com algum grau de DCF. A elevada frequência observada nos estudos realizados no Brasil transparece a ineficácia dos métodos de controle adotados pelos clubes de criação no país, provavelmente por falha nos critérios de seleção de reprodutores livres da DCF, que são baseados no MRC, preconizado pelo CBRV. A idade mínima de 24 meses para a realização do MRC é outro ponto falho nos programas de controle da DCF, tendo em vista que, nessa fase, os cães já estão maduros sexualmente e os criadores não esperam o resultado definitivo do exame radiográfico para introduzi-los nos programas de reprodução (Tôrres, 2003; Tôrres et al., 2005; Froes, 2011).

No presente estudo, a distribuição de animais displásicos de acordo com o gênero foi semelhante ao encontrado por Tôrres e colaboradores $\left(1999^{\mathrm{a}}\right)$, que mostrou machos e fêmeas da espécie canina acometidos em igual proporção (Tôrres et al., 1999').

A idade média dos cães avaliados foi de quatro anos e dez meses, o que permitiu a utilização do MRC como padrão ouro para o diagnóstico, tendo em vista que vários pesquisadores (Brass, 1989; Smith et al., 1993; Tôrres et al, 2005; Froes, 2011) demonstram que a eficácia dessa técnica varia entre 92 e 95\%, quando realizada após os dois anos de idade. A alta sensibilidade do MRC, quando realizado em animais adultos, é justificada pela utilização associada da aferição do AN e a avaliação dos sinais radiográficos da DAD, presentes nos animais displásicos nessa idade (Tôrres, 2003; Tôrres et al., 2007; Risler et al., 2009; Vanden Berg-Foels et al., 2011; Froes, 2011).

Tendo como referência o MRD, pode-se afirmar que 11 cães foram qualificados com ID normal. Entretanto, sete desses cães considerados normais, quando avaliados pelo MRD, foram classificados como displásicos por meio do MRC (Tab. 1), e isso indica que são resultados falsos negativos. Também é possível verificar na mesma tabela que os animais com resultados discordantes têm idade acima dos 30 meses. Esse resultado pode ser explicado por uma menor complacência das estruturas articulares nos cães adultos e doentes, fato que impede uma distensão completa da cápsula articular e, por consequência, reduz a eficácia do MRD nessa idade (Tôrres, 2003; Ginja et al., 2009).

Tabela 1. Distribuição dos resultados do diagnóstico da Displasia Coxofemoral, por meio do MRC e MRD, comparados com a idade, de 16 cães da raça Pastor Alemão adultos

\begin{tabular}{cccc}
\hline $\mathrm{N}^{0}$ Animal & Diagnóstico DCF - MRC & Diagnóstico DCF - MRD & Idade (anos) \\
\hline 1 & DCF Média & Normal* & 8,40 \\
2 & Normal & Normal & 7,50 \\
3 & Suspeito & Normal & 3,10 \\
4 & DCF Leve & Normal* & 10,96 \\
5 & DCF Leve & Displásico & 2,35 \\
6 & DCF Leve & Normal* & 3,66 \\
7 & DCF Leve & Displásico & 4,63 \\
8 & DCF Leve & Normal* & 7,24 \\
9 & DCF Leve & Normal* & 3,16 \\
10 & DCF Média & Normal* & 4,88 \\
11 & DCF Leve & Displásico & 7,24 \\
12 & DCF Leve & Normal* & 2,92 \\
13 & Normal & Normal & 2,68 \\
14 & Normal & Normal & 2,39 \\
15 & DCF Grave & Displásico & 4,00 \\
16 & DCF Leve & Displásico & 2,00 \\
\hline
\end{tabular}

*Animais que apresentaram resultados discordantes na avaliação por meio do MRC (método radiográfico convencional) e MRD (método radiográfico em distração). 
A Tabela 2 apresenta os resultados da ANOVA entre os diagnósticos do MRC e do MRD com a idade dos animais. Observa-se que, quando se utiliza o MRC como método diagnóstico, a média de idade dos cães displásicos é de 5,54 anos, ao passo que, quando se utiliza o MRD, a média de idade é menor, 4,05 anos.

Nota-se a existência de associação significativa entre a idade mais avançada dos cães e a presença de displasia pelo método do MRC, sendo que o mesmo não ocorre com o MRD.

Tal fato demonstra que, em animais adultos, o MRC apresenta uma sensibilidade maior que o MRD para o diagnóstico de DCF na medida em que a idade avança, concordando com Tôrres (2003).

Tabela 2. Resultados da análise de variância entre os diagnósticos do MRC e do MRD com a idade de cães da raça Pastor Alemão adultos

\begin{tabular}{lccc}
\hline Categoria & Idade Média & Desvio-padrão & $\mathrm{p}$ \\
\hline MRC - não displásico & 3,59 & 1,82 & $0,011^{*}$ \\
MRC - displásico & 5,54 & 2,75 & \\
\hline MRD - não displásico & 5,16 & 2,81 & 0,172 \\
MRD - displásico & 4,05 & 1,94 & \\
\hline *Valor-p $<0,05$ - MRC (método radiográfico convencional) e MRD (método radiográfico em distracão)
\end{tabular}

O estudo da correlação entre o AN e o ID mostrou-se significativo e negativo entre si e entre os lados esquerdo e direito, ou seja, quanto menor o AN, maior será o valor de ID (Tab. 3). Esse resultado é concordante com Smith e colaboradores (1990), Tôrres et al. (2005) e Ginja e colaboradores (2009). Entretanto, a correlação observada foi moderada e, possivelmente por isso, a utilização isolada do MRD tenha se mostrado pouco eficaz nos animais com mais de 30 meses de idade. Com valores de sensibilidade de $75 \%$ e especificidade de $44 \%$, reforçam o proposto por Froes (2011), que sugere a realização do MRC para animais adultos e somente nos casos de resultados negativos deve-se utilizar o MRD.

Tabela 3. Correlações entre as médias dos resultados do AN e ID, das articulações esquerda e direita, de 16 cães da raça Pastor Alemão adultos

\begin{tabular}{llcc}
\hline & & ID \\
\hline AN esquerda & $\mathrm{R}$ & $-0,379$ & $-0,478$ \\
& $\mathrm{p}$ & 0,008 & 0,001 \\
AN direita & $\mathrm{R}$ & $-0,573$ & $-0,627$ \\
& $\mathrm{p}$ & 0,000 & 0,000 \\
\hline
\end{tabular}

\section{CONCLUSÕES}

Com base nos resultados observados, concluiu-se que o MRC utilizado para diagnóstico da DCF, em cães da raça Pastor Alemão com idade superior a 30 meses, mostrou-se mais eficaz, com maiores sensibilidade e especificidade, quando comparado ao MRD. Apesar de o MRD ser mais eficaz na detecção da frouxidão articular passiva em animais com menos de 12 meses de idade, esse método não deve ser utilizado como primeira opção de diagnóstico da DCF em cães adultos.

\section{REFERÊNCIAS}

BRASS, W. Hip dysplasia in dogs. J. Sm. An. Prac., v.30, p.166-170, 1989.

BRASS, FREUDIGER, U.; MULLER, L.F; et al. Bericht der hüfpelenkdysplasie-kommission. Kleintier Praxis, v.23, p.169-180, 1978.

COLÉGIO Brasileiro De Radiologia Veterinária. Normas do Colégio. Avaliable from: <http://www.abrv.com.br/col_normas.php>. Cited: 09 Nov. 2007.

FROES, T.R. Diagnóstico radiográfico das doenças articulares pélvicas do cão. Rev. Vet. Zootec. Min. v.21, p.35-40, 2011. 
FROES, T.R.; GARCIA, D.A.A.; SCHMIDLIM, P.C. et al. Estudo comparativo e análise interobservador entre dois métodos de avaliação da displasia coxofemoral de cães. Arch. of Vet. Sci., v.14, p.187-197, 2009.

GINJA, M.M.; FERREIRA, A.J.; SILVESTRE, M. et al. Repeatability and reproducibility of distraction indices in PennHIP examinations of the hip joint in dogs. Acta Vet. Hung., v.57, p.387-392, 2006.

GINJA, M.M.; FERREIRA, A.J.; JESUS, S.S. et al. Comparison of clinical, radiographic, computed tomographic, and magnetic resonance imaging methods for early prediction of canine hip laxity and dysplasia. Vet. Radiol. Ultrasound., v.50, p.135-143, 2009.

LAFOUND, E.; BREUR, G.J.; AUSTIN, C.C. Breed susceptibility for developmental orthopedic diseases in dogs. J. Am. Hosp. Assoc., v.38, p.467-477, 2002.

MORGAN, J. Pathologic alterations in canine hip dysplasia. J. Am. Vet. Med. Assoc., v.210, p.1446-1450, 1997.

ORTHOPEDIC Foundation For Animals. Hip grades. Avaliable from: <http://www.offa.org/ hipgrade.html>. Cited: 19/02/2012.

RISLER, A.; KLAUER, J.M.; KEULER, N.S. et al. Puppy line, metaphyseal sclerosis, and caudolateral curvilinear and circumferential femoral head osteophytes in early detection of canine hip dysplasia. Vet. Radiol. Ultrasound, v.50, p.157-166, 2009.

SMITH, G.K.; LAWLER, D.F; BIERY, D.N. et al. Chronology of Hip Dysplasia Development in a Cohort of 48 Labrador Retrievers Followed for Life. Vet. Surg., v.41, p.20-33, 2012.

SMITH, G.K.; BIERY, D.M.; KEALY, R.D. et al. Significado clínico dos achados sobre osteoartrite e displasia coxofemoral no ensaio de restrição alimentar. Inf. Tec. Nestlé Purina, v.5, p.23-24, 2002.

SMITH, G.K.; BIERY, D.N.; GREGOR, T.P. New concepts of coxofemoral joint stability and the development of a clinical stress-radiographic method for quantitating hip joint laxity in the dog. J. An. Vet. Med. Assoc., v.196, p.59-70, 1990.
SOUZA, M.M.D.; PADOVANI, C.R.; RAHAL, S.C. et al. Afecções ortopédicas dos membros pélvicos em cães: estudo retrospectivo. Cienc. Rural, v.41, p.852-857, 2011.

TÔRRES, R.C.S. Prevalência da displasia coxofemoral em cães da raça Pastor Alemão, 1993. 80f. Dissertação (Mestrado em Ciência Animal) - Escola de Veterinária, Universidade Federal de Minas Gerais, Belo Horizonte.

TÔRRES, R.C.S. Uso do distrator articular no diagnóstico radiográfico precoce da displasia coxofemoral em cães, 2003. 70f. Tese (Doutorado em Ciência Animal) - Escola de Veterinária da Universidade Federal de Minas Gerais, Belo Horizonte.

TÔRRES, R.C.S.; ARAÚJO, R.B.; REZENDE, C.M.F. Distrator articular no diagnóstico radiográfico precoce da displasia coxofemoral em cães. Arq. Bras. Med. Vet. Zootec. v.57, p.2734, 2005.

TÔRRES, R.C.S.; OCARINO, N.M.; VIEIRA, G.L.T. et al. Aspectos patológicos e genéticos da displasia coxofemoral em cães. Rev. CFMV, v.13, p.27-32, 2007.

TÔRRES, R.C.S.; SILVA, E.F.; ROCHA, B.D. Displasia coxofemoral - frequência em cães da raça Rottweiler no Estado de Minas Gerais. Revista CFMV, v.9, p.60-64, 2003.

TÔRRES, R.C.S.; FERREIRA, P.M.; ARAÚJO, R.B. Presença de "Linha Morgam” como indicador de displasia coxofemoral em cães da raça Pastor Alemão. Arq. Bras. Med. Vet. Zootec. v.51, p.157-158, 1999a.

TORRES, R.C.S.; FERREIRA, P.M.; SILVA, D.C.. Frequência e assimetria da displasia coxofemoral em cães Pastor-Alemão. Arq. Bras. Med. Vet. Zootec., v.51, p.153-156, 1999 ${ }^{\mathrm{b}}$.

VANDEN BERG-FOELS, W.; SCHWAGER, S. et al. Femoral Head Bone Mineral Density Patterns May Identify Hips at Risk of Degeneration. Annals of Biom. Engineering. v.39, p.75-84, 2011.

VIEIRA, G.L.T.; TÔRRES, R.C.S.; BARROS, G.S. et al. Associação entre o ângulo de Norberg, o percentual de cobertura da cabeça femoral, o índice cortical e o ângulo de inclinação em cães com displasia coxofemoral. Arq. Bras. Med. Vet. Zootec., v.62, p.1094-1101, 2010. 